\title{
Entrance Time and Rényi Entropy
}

\author{
Chinmaya Gupta * Nicolai Haydn ${ }^{\dagger} \quad$ Milton Ko ${ }^{\ddagger} \quad$ Erika A. Rada-Mora $\S$
}

October 22, 2018

\begin{abstract}
For ergodic systems with generating partitions, the well known result of Ornstein and Weiss shows that the exponential growth rate of the recurrence time is almost surely equal to the metric entropy. Here we look at the exponential growth rate of entrance times, and show that it equals the entropy, where the convergence is in probability in the product measure. This is however under the assumptions that the limiting entrance times distribution exists almost surely. This condition looks natural in the light of an example by Shields in which the limsup in the exponential growth rate is infinite almost everywhere but where the limiting entrance times do not exist. We then also consider $\phi$-mixing systems and prove a result connecting the Rényi entropy to sums over the entrance times orbit segments.
\end{abstract}

\section{Introduction}

Let $T$ be a map on a space $\Omega$, then $\left\{T^{i}(x)\right\}_{i=0}^{\infty}$ defines the orbit of $x \in \Omega$. For a set $A \subset \Omega$, the entrance time $\tau_{A}$ of a point $x$ into the set $A$ refers to the time that takes for the orbit of $x$ to first enter the set $A$. In particular, if $x \in A, \tau_{A}$ refers to the return time of the point $x$ : the time that it takes the orbit of $x$ to return for the first time to the set $A$. For invariant probability measures $\mu$ the Poincaré Recurrence Theorem states that a point in a positive measure set returns to that set almost surely. In other words $\tau_{A}(x)<\infty$ for almost every $x$ in $A$, provided $\mu(A)>0$. In 1946 this result was quantified by Kac who showed that for ergodic measures the expected return time is the reciprocal of the measure of the return set. If the space $\Omega$ has a generating partition $\mathcal{A}$ then in 1993, Ornstein and Weiss 10 proved for ergodic measures $\mu$ that $\frac{1}{n} \log \tau_{n}$ converges to the entropy $h_{\mu}$ almost surely, where the $n$-th recurrence time $\tau_{n}(x)=\tau_{A_{n}(x)}(x)$ measures the time for $x$ to return to its initial $n$-cylinder $A_{n}(x)$.

Intuitively, the entrance time should behave similar to the return time in ergodic systems, as in such systems when a point $x$ travels long enough it tends to forget where it started. If we assume $\Omega$ has a partition $\mathcal{A}$, then it is natural to consider the exponential growth rate of entrance times to the $n$ cylinders $A_{n}(z)$ centred at an arbitrary point $z$. However, Shields 15 in 1992 constructed an example in which $\frac{1}{n} \log \tau_{A_{n}(z)}(x)$ does not converge for almost every $x$. In fact the limsup goes to infinity almost surely. Here we impose an additional assumption in order to get convergence in probability to the metric entropy. We require that the limiting entrance times distributions exist almost everywhere.

We then also give a condition under which the convergence of is almost surely. We then also look at $\phi$-mixing measures and show that they satisfy this conditions and thus have almost sure convergence of exponential growth rate of entrance times. In the last theorem we consider the Rényi entropy which was first introduced by Alfréd Rényi [13 in 1961 in order to generalize the Shannon entropy. Here we generalize a result of Ko 8 ] which had been proven for return times to entrance times. For $\phi$-mixing systems we obtain in Theorem 5 a relationship between entrance time and the Rényi entropy.

\footnotetext{
*Mathematics Department, University of Houston. E-mail: <ccgupta@math.uh.edu>.

${ }^{\dagger}$ Mathematics Department, USC, Los Angeles, 90089-1113. E-mail: < nhaydn@math.usc.edu>.

${ }_{\ddagger}$ Mathematics Department, USC, Los Angeles, 90089-1113. E-mail:<miltonko@gmail.com>.

§Institute of Mathematic and Statistic, University of São Paulo , 05508-090, Brazil. E-mail:<alejarada@gmail.com>. The fourth author was supported by CNPq-Brazil Procs. 143256/2009-2 and 140114/2012-2.
} 
In section 2 we state definitions, basic facts and the four main theorems that we will prove in this paper. Theorem 1 proves the convergence of the entrance time in probability while Theorem 2 proves the almost sure convergence of entrance time under an additional assumption. Theorem 4 verifies that additional assumption for $\phi$-mixing measures. Theorem 5 considers the sum of measures of $n$-cylinders visited by a point along its orbit until it enters a set, and proves that it converges to a constant in terms of the Rényi entropy and metric entropy for $\phi$-mixing systems. The proofs of Theorem 1 and 2 are given in Section 3, the proof of Theorem 4 is in section 4 while the proof of Theorem 5 is given in Section 5 .

\section{Main Results}

Let $\Omega$ be a space with a probability measure $\mu$ and $T: \Omega \rightarrow \Omega$ be a measurable map. We assume $\mu$ is $T$-invariant and ergodic. Let $\mathcal{A}=\left\{\mathcal{P}_{i}\right\}$ be a generating partition (finite or countably infinite) and denote by $\mathcal{A}^{n}=\bigvee_{i=0}^{n-1} T^{-i} \mathcal{A}=\left\{\bigcap_{0 \leq i \leq n-1} T^{-i}\left(\mathcal{P}_{j_{i}}\right): \mathcal{P}_{j_{i}} \in \mathcal{A}\right\}$ its $n$-th join. The elements of $\mathcal{A}^{n}$ are referred to as $n$-cylinders. We denote by $A_{n}(x) \in \mathcal{A}^{n}$ the $n$-cylinder which contains the point $x \in \Omega$.

The theorem of Shannon-McMillan-Breiman (see e.g. 9]) states that for any $T$-invariant ergodic probability measure $\mu$ and generating partition $\mathcal{A}$ of $\Omega$,

$$
\lim _{n \rightarrow \infty} \frac{1}{n}\left|\log \mu\left(A_{n}(x)\right)\right|=h_{\mu}
$$

for almost every $x \in \Omega$, where $h_{\mu}$ is the measure theoretic entropy of $\mu$. This asymptotic formula was first proven by Shannon [14 in 1948 for stationary Markov chains and then subsequently strengthened by McMillan and Breiman to its present form for finite alphabets and then extended to countably infinite alphabets (with finite entropy) by Chung [3] in 1961 and Carleson 2 in 1958. In other words, the measure of the $n$-cylinder which contains $x$ decays exponentially with rate roughly the metric entropy.

For any $x \in \Omega$ and set $A \subset \Omega$, let us define now the entrance time of $x$ into the set $A$ by

$$
\tau_{A}(x)=\min \left\{i \geq 1: T^{i}(x) \in A\right\} .
$$

We call $\tau_{n}(x)=\tau_{A_{n}(x)}(x)$ the $n$-th recurrence time of $x$; it is the first time that $x$ returns to the $n$ cylinder which contain $x$. Ornstein and Weiss proved in [10] for finite partition, and in [11] for countably infinite partition (provided that $h_{\mu}$ is finite) that for almost every $x$,

$$
\lim _{n \rightarrow \infty} \frac{1}{n} \log \tau_{n}(x)=h_{\mu}
$$

assuming $\mu$ is ergodic. Intuitively, the entrance time $\tau_{A_{n}(z)}(x)$ should behave similarly to the recurrence time $\tau_{n}(z)=\tau_{A_{n}(z)}(z)$ as in (2), since when points travel a long enough time in ergodic systems they tend to forget where they start and hence whether starting at the point $x$ or $z$ should not matter. However, Shields constructed in 1992 an example of a dynamical system in which the entrance time fails to converge [15]. Here we prove that $\frac{1}{n} \log \tau_{A_{n}(z)}(x)$ converges in probability to $h_{\mu}$ provided the system has an almost sure entrance times distribution.

In the following we adopt probability notations that for events $A, B \subset \Omega$ we denote $\mu(A)$ by $\mathbb{P}(A)$ and $\mu_{B}(A)=\mu(B \cap A) / \mu(B)$ by $\mathbb{P}_{B}(A)$ (assuming $\mu(B)>0$ ). For $z \in \Omega, n \in \mathbb{N}$ and $t>0$, put

$$
F_{z}^{n}(t)=\mathbb{P}\left(\tau_{A_{n}(z)} \geq \frac{t}{\mu\left(A_{n}(z)\right)}\right)=\mu\left(\left\{x \in \Omega: \tau_{A_{n}(z)}(x) \geq \frac{t}{\mu\left(A_{n}(z)\right)}\right\}\right)
$$

and if $B=A_{n}(z)$ we put

$$
F_{B}(t)=F_{z}^{n}(t) .
$$

We shall require that the $\operatorname{limit}_{\lim _{n \rightarrow \infty}} F_{z}^{n}$ exists almost everywhere. For a number of classes of positive entropy systems this limit is $e^{-t}$ a.s.. There are however examples of ergodic zero entropy systems that have other limiting distributions. 
The following two theorems prove convergences of the entrance time: Theorem1 1 proves the existence of the limit and convergence in probability under the assumption that the limiting entrance (or return) times exists almost everywhere. Theorem 2 gives a sufficient condition under which the convergence is almost sure. Let us note that there are many examples when the limiting entrance/return times do not exist. The example of Shields is one of them. Also, Downarowicz [5] has given examples when the limiting distribution exists along subsequences of full density and where the limit can to be made to decay arbitrarily slowly, in particular so slow as to violate the condition in Theorem 2.

Theorem 1. Suppose for almost every $z \in \Omega$ and for $t \geq 0, \lim _{n \rightarrow \infty} F_{z}^{n}(t)=F_{z}(t)$ exists and $F_{z}(t) \rightarrow 0$ as $t \rightarrow \infty$. Then $\frac{1}{n} \log \tau_{A_{n}(z)}(x)$ converges to $h_{\mu}$ in probability as $n$ goes to $\infty$.

Theorem 2. Suppose $\mu$ is a T-invariant ergodic probability measure on $\Omega$, and for all small enough $\epsilon>0$ we have

$$
\sum_{n=1}^{\infty} \int_{\Omega} F_{z}^{n}\left(e^{n \epsilon}\right) d \mu(z)<\infty
$$

Then

$$
\lim _{n \rightarrow \infty} \frac{1}{n} \log \tau_{A_{n}(z)}(x)=h_{\mu}
$$

for $\mu \times \mu$-almost every $(x, z) \in \Omega \times \Omega$.

Remark. (i) Let us note that summability condition of Theorem 2 is only required to get the upper bound on the limit. By Lemma 6 we get the lower bound on the limit almost surely for all ergodic measures.

(ii) Although the recurrence time $\tau_{n}(x)=\tau_{A_{n}(x)}(x)$ is a special case of the return time, Theorem 2 does not imply the asymptotic formula in (2) since the above convergence is true for $\mu \times \mu$-almost every $(x, z)$ which that does not imply that it applies to points on the diagonal $x=z$ as the diagonal has measure 0 in the product measure.

The remainder of the paper looks at a situation in which the hypothesis of Theorem 2 is satisfied. We consider systems with some mixing property.

Definition 3. We say an invariant measure $\mu$ is $\phi$-mixing if there exists a decreasing function $\phi: \mathbb{N} \rightarrow$ $\mathbb{R}$ so that

$$
\frac{\left|\mu\left(A \cap T^{-(n+i)}(B)\right)-\mu(A) \mu(B)\right|}{\mu(A)} \leq \phi(i)
$$

for all $A \in \mathcal{A}^{n}$, all $B \in \sigma\left(\mathcal{A}^{*}\right)$, where $\mathcal{A}^{*}=\cup_{n=1}^{\infty} \mathcal{A}^{n}$ and for all $n \in \mathbb{N}$.

In the following two theorems will moreover assume that $\phi$ is summable, that is $\sum_{i=1}^{\infty} \phi(i)<\infty$. Let us note that the limiting entrance times distribution $F_{z}(t)$ for $\phi$-mixing measures (with summable $\phi)$ is exponential almost everywhere [1, i.e. $F_{z}(t)=e^{-t}$ for $\mu$-almost every $z \in \Omega$. This includes in particular measures of maximal entropy and equilibrium states for Hölder continuous potential on Axiom A systems which are $\psi$-mixing at an exponential rate.

Theorem 4. Suppose $\mu$ is a T-invariant $\phi$-mixing measure of $\Omega$ with summable $\phi$. Then

$$
\lim _{n \rightarrow \infty} \frac{1}{n} \log \tau_{A_{n}(z)}(x)=h_{\mu}
$$

for $\mu \times \mu$-almost every $(x, z) \in \Omega \times \Omega$.

For the final result we will also require that the (countably infinite) partition $\mathcal{A}=\left\{\mathcal{P}_{i}\right\}_{i=1}^{\infty}$ has an exponentially decaying tail if

$$
\mu\left(\bigcup_{i \geq j} \mathcal{P}_{i}\right)=\mathcal{O}\left(\delta^{j}\right)
$$

for all $j$ and for some $\delta<1$. If $|\mathcal{A}|$ is finite then (4) is trivially satisfied. 
For $s>0$, put

$$
Z_{n}(s)=\sum_{A_{n} \in \mathcal{A}^{n}} \mu\left(A_{n}\right)^{1+s}
$$

and define the Rényi Entropy Function 13 on $(0, \infty)$ by

$$
R(s)=\lim _{n \rightarrow \infty} \frac{1}{s n}\left|\log Z_{n}(s)\right|
$$

if the limit exists. For larger values of $s$, the Rényi entropy is weighted towards highest probability events. Moreover for the value $s=0$, the Rényi entropy typically coincides with the Shannon entropy. The Rényi entropy exists as a uniform limit in weakly $\psi$-mixing systems [6] and a pointwise limit under weaker assumption 8 .

Theorem 5. Suppose $T: \Omega \rightarrow \Omega$ is measurable, $\mu$ is $T$ invariant and $\phi$-mixing with summable $\phi$, and $\mathcal{A}$ has exponential tails. Suppose the Rényi entropy $R(s)$ exists for $s>0$. Then for $\mu \times \mu$ every $(x, z) \in \Omega \times \Omega$,

$$
\lim _{n \rightarrow \infty} \frac{1}{n} \log \sum_{i=1}^{\tau_{A_{n}(z)}(x)} \mu\left(A_{n}\left(T^{i}(x)\right)\right)^{s}=h_{\mu}-s R(s) .
$$

This generalises a previous result of Ko 8 in which $z$ was assumed to be equal to $x$. Obviously, (3) ensures the ergodicity of $\mu$. Furthermore, (3) implies the exponential decay of cylinders and this ensures that the metric entropy $h_{\mu}$ is positive. The summability of $\phi$ is needed to estimate the variance of the hitting time function (see Section 5.2). The condition (4) in particular implies that $h_{\mu}$ is finite (See Lemma 4 of [8]). It also allows us to control the "tail" of the partition $\mathcal{A}^{n}$ in the proof of Lemma 14. From now on we will abbreviate $\tau_{A_{n}(z)}(x)$ by $\tau_{n}^{z}(x)$ for convenience.

\section{Convergence of Entrance Time}

We first prove the lower bound of Theorem 1 and 2 ,

Lemma 6. Suppose $\mu$ is a T-invariant ergodic probability measure of $\Omega$. Then

$$
\liminf _{n \rightarrow \infty} \frac{1}{n} \log \tau_{n}^{z}(x) \geq h_{\mu}
$$

for $\mu \times \mu$-almost every $(x, z) \in \Omega \times \Omega$.

Proof. Let $0<b<c<h_{\mu}$, and put

$$
\mathbb{E}_{n}=\left\{x: \tau_{n}^{z}(x) \leq e^{b n}\right\} .
$$

Note that $\mathbb{E}_{n}=\bigcup_{j=1}^{\left[e^{b n}\right]} T^{-j}\left(A_{n}(z)\right)$. Then we have,

$$
\mu\left(\mathbb{E}_{n}\right) \leq \sum_{j=1}^{\left[e^{b n}\right]} \mu\left(T^{-j}\left(A_{n}(z)\right)\right)=\mu\left(A_{n}(z)\right) e^{b n} .
$$

By (11), $\mu\left(A_{n}(z)\right) \leq e^{-n c}$ for almost every $z$. Therefore, $\mu\left(\mathbb{E}_{n}\right) \leq e^{-(c-b) n}$, summable on $n$. By the Borel Cantelli Lemma, for almost every $z, \mu\left(\lim \sup \mathbb{E}_{n}\right)=0$. In words, this implies that for almost every $z$, the set of initial conditions $x$ for which the return times to $A_{n}(z)$ are smaller than $e^{b n}$ infinitely often have $\mu$ measure 0 . This implies

$$
\liminf _{n \rightarrow \infty} \frac{1}{n} \log \tau_{n}^{z}(x) \geq h_{\mu}
$$

for almost every $x$. 
Remark. Note that in the proof above we showed that for any $\varepsilon>0$ and almost every $z$,

$$
\lim _{n \rightarrow \infty} \mu\left(\left\{x: \frac{1}{n} \log \tau_{n}^{z}(x) \leq h_{\mu}-\varepsilon\right\}\right)=0,
$$

which is equivalent to

$$
\lim _{n \rightarrow \infty} \mu \times \mu\left(\left\{(x, z): \frac{1}{n} \log \tau_{n}^{z}(x) \leq h_{\mu}-\varepsilon\right\}\right)=0 .
$$

To complete the proof of Theorem[1]and 2, we obtain the other side of the inequality in Lemma 6] under certain assumptions. One might have attempted to show this by only assuming that the measure $\mu$ is $T$-invariant and ergodic. However, Shields [15] constructed an example of a dynamical system (on a four-element subshift) in which

$$
\limsup _{n \rightarrow \infty} \frac{1}{n} \log \tau_{n}^{z}(x)=\infty
$$

for $\mu \times \mu$-almost every $(x, z) \in \Omega \times \Omega$.

Lemma 7. Suppose for all small enough $\epsilon>0$ and $\delta>0$,

$$
\lim _{n \rightarrow \infty} \mu\left(\left\{x \in \Omega: F_{z}^{n}\left(e^{n \epsilon}\right)>\delta\right\}\right)=0
$$

for almost every $z \in \Omega$. Then $\frac{1}{n} \log \tau_{n}^{z}(x)$ converges to $h_{\mu}$ in probability as $n \rightarrow \infty$.

Proof. Let $\delta>0, b>h_{\mu}$ and $\mathbb{D}_{n}=\left\{(x, z) \in \Omega \times \Omega: \tau_{n}^{z}(x)>e^{n b}\right\}$. We want to show that $\mu \times \mu\left(\mathbb{D}_{n}\right)$ is bounded from above by $\delta$ for large enough $n$. Further let $\epsilon \in\left(0, b-h_{\mu}\right)$ and $\delta^{\prime}=b-\left(h_{\mu}+\epsilon\right)$. Put

$$
\begin{gathered}
\Omega_{n}=\left\{z \in \Omega: F_{z}^{n}\left(e^{n \epsilon}\right) \leq \delta / 3\right\}, \\
\mathcal{S}_{n}=\left\{B \in \mathcal{A}_{n}: \mu(B) \geq e^{-n\left(h_{\mu}+\delta^{\prime}\right)}\right\}
\end{gathered}
$$

and

$$
S_{n}=\bigcup_{B \in \mathcal{S}_{n}} B
$$

By hypothesis and (11), we can choose $n$ large enough so that $\mu\left(\Omega_{n}^{c}\right)<\delta / 3$ and $\mu\left(S_{n}^{c}\right)<\delta / 3$. Put

$$
\bar{\Omega}_{n}=\left\{B \in \mathcal{A}^{n}: B \cap \Omega_{n} \neq \emptyset\right\}
$$

As $F_{z}^{n}(t)$ is locally constant on $n$-cylinders $B, F_{B}\left(e^{n \epsilon}\right) \leq \delta / 3$ for $B \in \bar{\Omega}_{n}$. Note also that $F_{B}(t)$ decreases as $t$ increases; therefore, $F_{B}\left(\mu(B) e^{n b}\right) \leq F_{B}\left(e^{n \epsilon}\right)$. It follows that for large enough $n$

$$
\begin{aligned}
\mu \times \mu\left(\mathbb{D}_{n}\right) & =\sum_{B \in \mathcal{A}^{n}} \mu(B) \mathbb{P}\left(\tau_{B} \geq e^{n b}\right) \\
& =\sum_{B \in \mathcal{A}^{n}} \mu(B) F_{B}\left(\mu(B) e^{n b}\right) \\
& =\sum_{B \in \bar{\Omega}_{n}^{c}} \mu(B) F_{B}\left(\mu(B) e^{n b}\right)+\sum_{B \in \bar{\Omega}_{n}} \mu(B) F_{B}\left(\mu(B) e^{n b}\right) \\
& <\mu\left(\Omega_{n}^{c}\right)+\sum_{B \in \mathcal{S}_{n}^{c}} \mu(B) F_{B}\left(\mu(B) e^{n b}\right)+\sum_{B \in \bar{\Omega}_{n} \cap \mathcal{S}_{n}} \mu(B) F_{B}\left(\mu(B) e^{n b}\right) \\
& <\frac{\delta}{3}+\mu\left(S_{n}^{c}\right)+\sum_{B \in \bar{\Omega}_{n} \cap \mathcal{S}_{n}} \mu(B) F_{B}\left(e^{n \epsilon}\right) \sum_{B \in \bar{\Omega}_{n} \cap \mathcal{S}_{n}} \mu(B) \leq \delta
\end{aligned}
$$


As the above is true for any $b>h_{\mu}$, we showed for any $\varepsilon>0$

$$
\lim _{n \rightarrow \infty} \mu \times \mu\left(\left\{(x, z): \frac{1}{n} \log \tau_{n}^{z}(x) \geq h_{\mu}+\varepsilon\right\}\right)=0 .
$$

Together with the remark under Lemma 6, the proof is completed.

Similar to the entrance time distribution, for $z \in \Omega, n \in \mathbb{N}$ and $t>0$ we define the return time distribution as

$$
\tilde{F}_{z}^{n}(t)=\mathbb{P}_{A_{n}(z)}\left(\tau_{A_{n}(z)} \geq \frac{t}{\mu\left(A_{n}(z)\right)}\right)=\mu\left(\left\{x \in A_{n}(z): \tau_{n}^{z}(x) \geq \frac{t}{\mu\left(A_{n}(z)\right)}\right\}\right) / \mu\left(A_{n}(z)\right)
$$

assuming $\mu\left(A_{n}(z)\right)>0$ and if $B=A_{n}(z)$ we put

$$
\tilde{F}_{B}(t)=\tilde{F}_{z}^{n}(t) .
$$

By [7] the entrance times distribution $F_{B}$ and the return times distribution $\tilde{F}_{B}$ are related by the identity $F_{B}(t)=\int_{t}^{\infty} \tilde{F}_{B}(s) d s$.

Proof of Theorem 1, Let $\beta, \epsilon$ and $\delta$ be positive. By Lemma 7 , we want to show that

$$
\lim _{n \rightarrow \infty} \mu\left(\left\{x \in \Omega: F_{z}^{n}\left(e^{n \epsilon}\right)>\delta\right\}\right)=0 .
$$

Put $V_{N}=\left\{z \in \Omega: F_{z}(N) \leq \delta / 2\right\}$. Since $F_{z}(t)$ decreases to 0 by assumption, there exists $K=N_{\delta, \beta}$ such that $\mu\left(V_{K}^{c}\right)<\beta / 2$. Put $U_{n}=\left\{z \in \Omega:\left|F_{z}^{n}(K)-F_{z}(K)\right| \leq \delta / 2\right\}$. Since $F_{z}^{n}$ converges to $F_{z}$ for almost every $z$, when $n$ is large enough, we have $\mu\left(U_{n}^{c}\right)<\beta / 2$, and $e^{n \epsilon}>K$. For $z \in V_{K} \cap U_{n}$, we get

$$
F_{z}^{n}\left(e^{n \epsilon}\right) \leq F_{z}^{n}(K) \leq F_{z}(K)+\delta / 2<\delta .
$$

This shows for large $n$,

$$
\mu\left(z \in \Omega: F_{z}^{n}\left(e^{n \epsilon}\right)>\delta\right) \leq \mu\left(V_{K}\right)+\mu\left(U_{n}\right)<\beta / 2+\beta / 2=\beta,
$$

and the proof is completed.

Now we turn to prove the almost sure convergence of the entrance time.

Proof of Theorem 2, Let $b>h_{\mu}, \epsilon \in\left(0, b-h_{\mu}\right)$ and $\delta=b-\left(h_{\mu}+\epsilon\right)$. We claim that

$$
\limsup _{n \rightarrow \infty} \frac{1}{n} \log \tau_{n}^{z}(x) \leq h_{\mu}
$$

for $\mu \times \mu$-almost every $(x, z) \in \Omega \times \Omega$. Put

$$
\mathcal{S}_{n}=\left\{B \in \mathcal{A}_{n}: \mu(B) \geq e^{-n\left(h_{\mu}+\delta\right)}\right\}
$$

and

$$
S_{n}=\bigcup_{B \in \mathcal{S}_{n}} B
$$

Then as $F_{B}(t)$ is decreasing, if we put $\mathbb{D}_{n}=\left\{(x, z) \in \Omega \times \Omega: \tau_{n}^{z}(x)>e^{n b}\right\}$, we have

$$
\begin{aligned}
\mu \times \mu\left(\mathbb{D}_{n} \cap\left(\Omega \times S_{n}\right)\right) & =\sum_{B \in \mathcal{S}_{n}} \mu(B) F_{B}\left(\mu(B) e^{n b}\right) \\
& \leq \sum_{B \in \mathcal{S}_{n}} \mu(B) F_{B}\left(e^{-n\left(h_{\mu}+\delta\right)} e^{n b}\right) \\
& =\sum_{B \in \mathcal{S}_{n}} \mu(B) F_{B}\left(e^{n \epsilon}\right)=\int_{\Omega} F_{z}^{n}\left(e^{n \epsilon}\right) d \mu(z)
\end{aligned}
$$


which is summable by our hypothesis. Applying the Borel Cantelli Lemma and (10) gives

$$
\mathbb{P}\left(\limsup _{n \rightarrow \infty} \mathbb{D}_{n}\right) \leq \mathbb{P}\left(\limsup _{n \rightarrow \infty}\left(\mathbb{D}_{n} \cap\left(\Omega \times S_{n}\right)\right)\right)+\mathbb{P}\left(\limsup _{n \rightarrow \infty}\left(\Omega \times S_{n}^{c}\right)\right)=0 .
$$

As $b>h_{\mu}$ is arbitrary our claim is proved. Together with Lemma 6, we proved Theorem 2 ,

Corollary 8. Suppose for almost every $z \in \Omega$, there exists $F_{z}(t)$, a decreasing function on $t>0$, and a summable sequence $a_{n}>0$ such that for all small enough $\epsilon>0$,

(i) $\sum_{n=1}^{\infty} \mu\left(\left\{z:\left|F_{z}^{n}\left(e^{n \epsilon}\right)-F_{z}\left(e^{n \epsilon}\right)\right|>a_{n}\right\}\right)<\infty$ and

(ii) $\sum_{n=1}^{\infty} \int_{\Omega} F_{z}\left(e^{n \epsilon}\right) d \mu(z)<\infty$.

Then

$$
\lim _{n \rightarrow \infty} \frac{1}{n} \log \tau_{n}^{z}(x)=h_{\mu}
$$

for $\mu \times \mu$-almost every $(x, z) \in \Omega \times \Omega$.

Proof. In light of Theorem 2 it is sufficient to show that our hypothesis implies

$$
\sum_{n=1}^{\infty} \int_{\Omega} F_{z}^{n}\left(e^{n \epsilon}\right) d \mu(z)<\infty
$$

for small enough $\epsilon$. But

$$
\begin{aligned}
& \int_{\Omega} F_{z}^{n}\left(e^{n \epsilon}\right) d \mu(z) \\
\leq & \int_{\Omega}\left|F_{z}^{n}\left(e^{n \epsilon}\right)-F_{z}\left(e^{n \epsilon}\right)\right| d \mu(z)+\int_{\Omega} F_{z}\left(e^{n \epsilon}\right) d \mu(z) \\
\leq & 2 \mu\left(\left\{z:\left|F_{z}^{n}\left(e^{n \epsilon}\right)-F_{z}\left(e^{n \epsilon}\right)\right|>a_{n}\right\}\right)+a_{n}+\int_{\Omega} F_{z}\left(e^{n \epsilon}\right) d \mu(z) .
\end{aligned}
$$

The three terms on the right hand side above are all summable by our hypothesis, and we are done.

\section{Proof of Theorem 4}

We shall need the following result of Abadi [1, Theorem 1]). The following is a simplified version.

Lemma 9. Let $\mu$ be a $\phi$-mixing $T$-invariant probability measure such that $\phi$ is summable. Then there exist a constants $M>0, K_{[}<\infty$ such that

$$
\mathbb{P}\left(\tau_{A}>\frac{t}{\mu(A)}\right) \leq e^{-M t}+K_{9}(n \mu(A)+\phi(n))
$$

for all $A \in \mathcal{A}^{n}$ and all $n \in \mathbb{N}$.

Proof of Theorem 4, We have to prove that the limit

$$
\lim _{n \rightarrow \infty} \frac{1}{n} \log \tau_{n}^{z}(x)=h_{\mu}
$$

exists for $\mu \times \mu$-almost every $(x, z) \in \Omega \times \Omega$ under the assumption that $\mu$ is $\phi$-mixing and $\sum_{i} \phi(i)<\infty$. By Theorem 2, we need to show that

$$
\sum_{n=1}^{\infty} \int_{\Omega} F_{z}^{n}\left(e^{n \epsilon}\right) d \mu(z)<\infty
$$


for any small enough $\epsilon$. It is well known that for a $\phi$-mixing system, there exists $r>0$ such that $\mu(A) \leq e^{-r n}$ for all $n$ and $n$-cylinder $A \in \mathcal{A}^{n}$. Moreover by Lemma 9 we have

$$
\mathbb{P}\left(\tau_{A_{n}(z)}>\frac{t}{\mu\left(A_{n}(z)\right)}\right) \leq e^{-M t}+K_{9}\left(n \mu\left(A_{n}(z)\right)+\phi(n)\right)
$$

for every $z \in \Omega, n \in \mathbb{N}$ and $t>0$. Then for any $\epsilon>0$,

$$
\begin{aligned}
\sum_{n=1}^{\infty} \int_{\Omega} F_{z}^{n}\left(e^{n \epsilon}\right) d \mu(z) & =\int_{\Omega} \sum_{n=1}^{\infty} \mathbb{P}\left(\tau_{A_{n}(z)}>\frac{e^{n \epsilon}}{\mu\left(A_{n}(z)\right)}\right) d \mu(z) \\
& \leq \int_{\Omega}\left\{e^{-M \exp (n \epsilon)}+K\left[{ }^{\mu} \mu\left(A_{n}(z)\right)+K[9(n)\} d \mu(z)\right.\right. \\
& \leq \int_{\Omega}\left\{e^{-M \exp (n \epsilon)}+K\left[e^{-r n}+K[9(n)\} d \mu(z)<\infty\right.\right.
\end{aligned}
$$

as required.

\section{Proof of Theorem 5}

From now on we will assume that the measure $\mu$ satisfies the $\phi$-mixing property with summable $\phi$ and the partition $\mathcal{A}$ has an exponentially decaying tail (see (3) and (4)). We separately prove the upper and lower bound on the limit. The upper is quite easy but the lower bound requires a more careful analysis of hitting numbers. For $z \in \Omega$, define

$$
D^{z}:=\left\{x: \lim _{n \rightarrow \infty} \frac{1}{n} \log \tau_{n}^{z}(x)=h_{\mu}\right\}
$$

and put

$$
D:=\left\{z: \mu\left(D^{z}\right)=1\right\}
$$

Theorem 4 implies that $\mu(D)=1$. For $z \in D, \epsilon>0$ and all $x \in D^{z}$, we have

$$
e^{n\left(h_{\mu}-\epsilon\right)}<\tau_{n}^{z}(x)<e^{n\left(h_{\mu}+\epsilon\right)}
$$

for large enough $n$. In the rest of the paper we assume $z \in D$, and for convenience we put $W_{n}^{s}(x, z)=$ $\sum_{i=1}^{\tau_{n}^{z}(x)} \mu\left(A_{n}\left(T^{i}(x)\right)\right)^{s}$. Note that $W_{n}^{0}(x, x)=\tau_{n}(x)$, and in this case Theorem 5 coincides with (2). Also the case $x=z$ and $s>0$ of Theorem 5 was proven in [8].

\subsection{Proof of the upper bound of the limit in Theorem 5}

By the proof of Proposition 2.3 in [4] (see also [8] Proposition 6) for every $\epsilon>0$ there exists $D_{\epsilon} \subset \Omega$ with measure 1 such that for $x \in D_{\epsilon}$,

$$
\limsup _{n \rightarrow \infty} \frac{1}{n} \log \sum_{i=1}^{\exp \left(n\left(h_{\mu}+\frac{\epsilon}{3}\right)\right)} \mu\left(A_{n}\left(T^{i}(x)\right)\right)^{s} \leq h_{\mu}-s R(s)+\epsilon .
$$

Also by (5), we know that for $z \in D$ and therefore for all $x \in D^{z} \cap D_{\epsilon}$,

$$
\limsup _{n \rightarrow \infty} \frac{1}{n} \log W_{n}^{s}(x, z) \leq \limsup _{n \rightarrow \infty} \frac{1}{n} \log \sum_{i=1}^{\exp \left(n\left(h_{\mu}+\frac{\epsilon}{3}\right)\right)} \mu\left(A_{n}\left(T^{i}(x)\right)\right)^{s} \leq h_{\mu}-s R(s)+\epsilon .
$$

Finally, as $\mu\left(\bigcap_{m=1}^{\infty} D_{1 / m} \cap D^{z}\right)=1$, this establishes the upper bound in Theorem 5 . 


\section{$5.2 \quad$ Hitting numbers}

To prove the lower bound on the limit in Theorem 5 we need estimates on the hitting number

$$
N_{U, M}(x)=\sum_{i=0}^{M} \chi_{U} \circ T^{i}(x)
$$

of $U \in \sigma\left(\mathcal{A}^{n}\right)$ (unions of $n$-cylinders), where $\chi_{U}$ is the characteristic function of the set $U . N_{U, M}(x)$ counts the number of times $i \in[0, M]$ that $T^{i}(x) \in U$. Similarly $\nu_{x}^{z}(U)=N_{U, \tau_{n}^{z}(x)}(x)$ is the number of times that $x$ hits the set $U$ when it travels along its orbit segment until it returns to $A_{n}(z)$. Following [4] it was shown in [8] that the variance of the hitting time can be estimated by $\operatorname{Var}\left(N_{U, M}\right) \leq c_{1} M n \mu(U)$ for a constant $c_{1}$.

The following two lemmas provide us with lower and upper bounds for the hitting time. For $z=x$ these results have been proven in [8] and here we give the modification required for the present more general setting.

Lemma 10. Let $\mu$ be an $\phi$-mixing T-invariant measure where $\phi(i)$ is summable and $U_{n} \in \sigma\left(\mathcal{A}^{n}\right)$, $n=1,2, \ldots$, be a sequence of sets in $\sigma\left(\mathcal{A}^{n}\right)$. Let $\epsilon>0$ and assume $\gamma_{n}$ is a sequence of positive numbers so that for all $n$ large enough $(C, a, b>0$ constants): Assume one of the following two conditions are satisfied:

(I) $\mu\left(U_{n}\right) \geq C e^{-\gamma_{n}\left(h_{\mu}+\epsilon\right)}$ and $\left(n-\gamma_{n}\right) h_{\mu}-\epsilon\left(n+\gamma_{n}\right) \geq a n^{b}$,

(II) $\mu\left(U_{n}\right) \geq C e^{-\gamma_{n}\left(h_{\mu}-\epsilon\right)}$ and $\left(n-\gamma_{n}\right)\left(h_{\mu}-\epsilon\right) \geq a n^{b}$.

Then for almost every $(x, z)$,

$$
N_{U_{n}, \tau_{n}^{z}(x)}(x) \geq \frac{\mu\left(U_{n}\right)}{2} e^{n\left(h_{\mu}-\epsilon\right)}
$$

for all $n$ large enough.

Proof. (I) Put $M=\left[e^{n\left(h_{\mu}-\epsilon\right)}\right]$. Using the estimate on the variance of $N_{U_{n}, M}$ and Chebycheff's inequality it was shown in $\left[8\right.$, that there exists a set $D^{\prime}$, with measure 1 , such that, for all $x \in D^{\prime}$, and for all $n$ large enough, it holds, $\frac{N_{U_{n}, M}(x)}{M} \geq \mu\left(U_{n}\right) / 2$. Since by (5) for $z \in D, x \in D^{z} \cap D^{\prime}$, and for $n$ large enough, we have $\tau_{n}^{z}(x)>e^{n\left(h_{\mu}-\epsilon\right)}$, and therefore

$$
N_{U_{n}, \tau_{n}^{z}(x)}(x) \geq N_{U_{n}, M}(x) \geq M \mu\left(U_{n}\right) / 2=\mu\left(U_{n}\right) e^{n\left(h_{\mu}-\epsilon\right)} / 2 .
$$

Since $\mu\left(D^{z} \cap D^{\prime}\right)=1$, the estimate follows.

Part (II) is proven similarly.

Lemma 11. Let $\mu$ be as in Lemma 10 and $U_{n} \in \sigma\left(\mathcal{A}^{n}\right), n=1,2, \ldots$, be a sequence of sets. Suppose there exists a constant $C>0$ so that $\mu\left(U_{n}\right) \geq C$ for all large enough $n$. Then for $\epsilon>0$ and for almost every $x$,

$$
N_{U_{n}, \tau_{n}^{z}(x)}(x) \leq \frac{3 \mu\left(U_{n}\right)}{2} e^{n\left(h_{\mu}+\epsilon\right)}
$$

for all $n$ large enough.

Proof. For $M=\left[e^{n\left(h_{\mu}+\epsilon\right)}\right]$ it was shown in $\left[8\right.$ that $\left|\frac{N_{U_{n}, M}(x)}{M}-\mu\left(U_{n}\right)\right| \leq \mu\left(U_{n}\right) / 2$ for all $n$ large enough. By (5) for $z \in D, x \in D^{z} \cap D^{\prime}$, and for large enough $n$, we have $\tau_{n}^{z}(x)<e^{n\left(h_{\mu}+\epsilon\right)}$, and hence $N_{U_{n}, \tau_{n}^{z}(x)}(x) \leq N_{U_{n}, M}(x) \leq 3 M \mu\left(U_{n}\right) / 2=3 \mu\left(U_{n}\right) e^{n\left(h_{\mu}+\epsilon\right)} / 2$ as desired.

Using (11) and Egoroff's Theorem, there exists a set $\mathcal{E}$ with measure greater than $1 / 2$ on which $\left|\log \mu\left(A_{n}(x)\right)\right| / n$ converges to $h_{\mu}$ uniformly as $n \rightarrow \infty$. Define

$$
E_{n}:=\left\{x: A_{n}(x) \cap \mathcal{E} \neq \emptyset\right\},
$$

the union of those $n$-cylinders which intersect $\mathcal{E}$. As $E_{n} \in \sigma\left(\mathcal{A}^{n}\right)$, let us apply Lemma 10 and 11 to obtain estimations on the hitting number of $E_{n}$. 
Corollary 12. For any positive $\epsilon<h_{\mu}$ and almost every $(x, z)$,

$$
\begin{aligned}
& \text { (I) } \nu_{x}^{z}\left(E_{n}\right) \geq \frac{\mu\left(E_{n}\right)}{2} e^{n\left(h_{\mu}-\epsilon\right)} ; \\
& \text { (II) } \nu_{x}^{z}\left(E_{n}^{c}\right) \leq \frac{3 \mu\left(E_{n}^{c}\right)}{2} e^{n\left(h_{\mu}+\epsilon\right)}
\end{aligned}
$$

for all $n$ large enough (where $\nu_{x}^{z}(U)=N_{U, \tau_{n}^{z}(x)}(x)$ ).

Proof. (I) We use Lemma 10(II) with $U_{n}=E_{n}$ and $\gamma_{n}=n / 2$ for any $a, b>0$. In order to verify the second part of the condition of Lemma 10(II) note that $\mu\left(E_{n}\right) \geq \mu(\mathcal{E}) \geq 1 / 2$ for all $n$. Thus $\mu\left(E_{n}\right) \geq e^{-\gamma_{n}\left(h_{\mu}-\epsilon\right)}$ for all large enough $n$ and Lemma 10(II) gives the desired result. (II) First suppose that $\mu\left(E_{m}\right)<1$ for some integer $m$. Since $E_{n+1} \subseteq E_{n}$ (as $A_{n+1}(x) \subseteq A_{n}(x)$ ) we conclude that $\mu\left(E_{n}^{c}\right) \geq \mu\left(E_{m}^{c}\right)$ for all $n>m$. Hence by Lemma 11 (with $C=\mu\left(E_{m}^{c}\right)$ ) we are done. If $\mu\left(E_{n}\right)=1$ for all $n$, then put

$$
B=\bigcap_{n \geq 1} \bigcap_{i \geq 0} T^{-i}\left(E_{n}\right)
$$

Then $\mu(B)=1$ and for $x \in B, T^{i}(x) \notin E_{n}^{c}$ for any $n$. Hence for almost every $x, \nu_{x}^{z}\left(E_{n}^{c}\right)=0=$ $\frac{3 \mu\left(E_{n}^{c}\right)}{2} e^{n\left(h_{\mu}+\epsilon\right)}$ for all $n$.

\subsection{Proof of the lower bound of the limit in Theorem 5}

Let $c, \alpha \in(0,1)$, and $\epsilon>0$ be a small number which depends on $h_{\mu}$ and $c$ (which is close to 1), and will be determined later. Define $\tilde{\gamma}_{n}=n-\left[n^{\alpha}\right]$ and $\Delta=\left[n^{\alpha}\right]$. Denote by $\tilde{A}_{n}(x) \in \mathcal{A}^{\tilde{\gamma}_{n}}$ and $\bar{A}_{n}(x) \in \mathcal{A}^{[c n]}$ the $\tilde{\gamma}_{n}$-cylinder and the $[c n]$-cylinder which contain $x$ respectively. As $n>\tilde{\gamma}_{n}>c n$ (for $n$ large enough), we have $A_{n}(x) \subset \bar{A}_{n}(x) \subset \tilde{A}_{n}(x)$. In the following we denote an $n$-cylinder by $A_{n}$ or $A$, a $[c n]$-cylinder by $\bar{A}_{n}$ or $\bar{A}$, and a $\tilde{\gamma}_{n}$-cylinder by $\tilde{A}_{n}$ or $\tilde{A}$. For $\epsilon>0$, there exists $K_{\epsilon}$ such that for any $n>K_{\epsilon}$, we have

$$
e^{-n\left(h_{\mu}+\epsilon\right)} \leq \mu\left(A_{n}(x)\right) \leq e^{-n\left(h_{\mu}-\epsilon\right)}
$$

for $x \in \mathcal{E}$. Note that for $n>K_{\epsilon}$ and if the $n$-cylinder $A \subset E_{n}$, we have $A=A_{n}(x)$ for some $x \in \mathcal{E}$ and hence $\mu(A)$ satisfies (6). From now on, we assume $n$ is large enough so that $n>\tilde{\gamma}_{n}>c n>K_{\epsilon}$ and hence (6) holds with $n$ replaced by $\tilde{\gamma}_{n}$ and $c n$. The inequality (6) shows the uniformity property of the measures of cylinders in the sense that when $x \in \mathcal{E}$, we have

$$
\mu\left(\bar{A}_{n}(x)\right) \leq e^{-c n\left(h_{\mu}-\epsilon\right)} \leq \mu\left(A_{n}(x)\right) e^{(1-c) n h_{\mu}+2 n \epsilon} .
$$

If we put

$$
\bar{E}_{n}=\left\{x: \bar{A}_{n}(x) \cap \mathcal{E} \neq \emptyset\right\},
$$

(the union of $[c n]$-cylinders which intersect $\mathcal{E}$ ) then $E_{n} \subseteq \bar{E}_{n} \subseteq \mathcal{E}$.

Let $\varepsilon=\frac{1-c}{1+c} h_{\mu}$ (recall that $h_{\mu}$ is positive, by the comment after (4)), and from now on we choose $\epsilon<\varepsilon$. When we let $\varepsilon \rightarrow 0$, we have both $\epsilon \rightarrow 0$ and $c \rightarrow 1$. For convenience we also put

$$
\begin{aligned}
& \tilde{E}_{n}^{+}=\left\{\tilde{A} \in \mathcal{A}^{\tilde{\gamma}_{n}}: \mu(\tilde{A}) \geq e^{-\tilde{\gamma}_{n}\left(h_{\mu}-\epsilon\right)}\right\} ; \\
& \tilde{E}_{n}^{-}=\left\{\tilde{A} \in \mathcal{A}^{\tilde{\gamma}_{n}}: \mu(\tilde{A}) \leq e^{-\tilde{\gamma}_{n}\left(h_{\mu}-\epsilon\right)}\right\} .
\end{aligned}
$$

According to 8 , the lower bound on the limit in Theorem 5 follows immediately from the following two lemmas.

Lemma 13. There exists some constant $K_{13}$, which depends only on $s$, so that for almost every $x$,

$$
W_{n}^{s}(x, z) \geq e^{\mathrm{K}} \overline{13}^{n \varepsilon} e^{n h_{\mu}} \sum_{\tilde{A} \in \tilde{E}_{n}^{-}} \mu(\tilde{A})^{1+s}
$$

for all $n$ large enough. 
Proof. We proceed in three steps:

(I) We have

$$
W_{n}^{s}(x, z) \geq \sum_{i=1}^{\tau_{n}^{z}(x)} \mu\left(A_{n}\left(T^{i}(x)\right)\right)^{s} \chi_{E_{n}}\left(T^{i}(x)\right) \geq e^{s\left((c-1) n h_{\mu}-2 n \epsilon\right)} S_{1} \geq e^{-4 s n \varepsilon} S_{1}
$$

as $\epsilon, 1-c<\varepsilon$, where $S_{1}=\sum_{i=1}^{\tau_{n}^{z}(x)} \mu\left(\bar{A}_{n}\left(T^{i}(x)\right)\right)^{s} \chi_{E_{n}}\left(T^{i}(x)\right)$.

(II) Put $S_{2}=\sum_{i=1}^{\tau_{n}^{z}(x)} \mu\left(\bar{A}_{n}\left(T^{i}(x)\right)\right)^{s} \chi_{\bar{E}_{n}}\left(T^{i}(x)\right)$. Then $S_{1} \leq S_{2}$ as $E_{n} \subseteq \bar{E}_{n}$. If $T^{i}(x) \in E_{n}$, then by (6) we have $\mu\left(\bar{A}_{n}\left(T^{i}(x)\right)\right) \geq e^{-c n\left(h_{\mu}+\epsilon\right)}$ and consequently by Corollary 12(I) $\left(\right.$ as $\mu\left(\bar{A}_{n}\left(T^{i}(x)\right)\right) \geq e^{-n\left(h_{\mu}+\epsilon\right)}$ for all $\left.x \in E_{n}\right)$

$$
S_{1} \geq \sum_{i=1}^{\tau_{n}^{z}(x)} e^{-s c n\left(h_{\mu}+\epsilon\right)} \chi_{E_{n}}\left(T^{i}(x)\right)=\nu_{x}^{z}\left(E_{n}\right) e^{-s c n\left(h_{\mu}+\epsilon\right)} \geq \frac{\mu\left(E_{n}\right)}{2} e^{n\left(h_{\mu}-\epsilon\right)} e^{-s c n\left(h_{\mu}+\epsilon\right)} .
$$

Meanwhile, by Corollary 12(II) (as $\mu\left(\bar{A}_{n}\left(T^{i}(x)\right)\right) \leq e^{-n\left(h_{\mu}-\epsilon\right)}$ for all $x \in E_{n}$ ) we also have

$$
\begin{aligned}
S_{2}-S_{1} & =\sum_{i=1}^{\tau_{n}^{z}(x)} \mu\left(\bar{A}_{n}\left(T^{i}(x)\right)\right)^{s} \chi_{\bar{E}_{n} \backslash E_{n}}\left(T^{i}(x)\right) \\
& \leq \sum_{i=1}^{\tau_{n}^{z}(x)} e^{-s c n\left(h_{\mu}-\epsilon\right)} \chi_{E_{n}}\left(T^{i}(x)\right) \\
& =\nu_{x}^{z}\left(E_{n}^{c}\right) e^{-s c n\left(h_{\mu}-\epsilon\right)} \\
& \leq \frac{3 \mu\left(E_{n}^{c}\right)}{2} e^{n\left(h_{\mu}+\epsilon\right)} e^{-s c n\left(h_{\mu}-\epsilon\right)}
\end{aligned}
$$

Since $\mu\left(E_{n}{ }^{c}\right)<1 / 2<\mu\left(E_{n}\right)$, we get

$$
S_{2}-S_{1} \leq 3\left[\frac{1}{2} \mu\left(E_{n}\right) e^{n\left(h_{\mu}-\epsilon\right)} e^{-s c n\left(h_{\mu}+\epsilon\right)}\right] e^{2 n \epsilon} e^{2 s c n \epsilon} .
$$

By (8) the quantity in the bracket of the above inequality is less than $S_{1}$. Consequently $S_{2}-S_{1} \leq$ $3 e^{2 n \epsilon(1+s c)} S_{1}$ and

$$
S_{2} \leq e^{4 n \epsilon(1+s c)} S_{1} \leq e^{4(1+s) \varepsilon n} S_{1}
$$

as $c<1$ and $\epsilon<\varepsilon$.

(III) We also have

$$
S_{2}=\sum_{\bar{A} \subset \bar{E}_{n}} \nu_{x}^{z}(\bar{A}) \mu(\bar{A})^{s}
$$

using the counting function $\nu_{x}^{z}(\bar{A})$ for which we have bounds by Lemma 10(I): since for $\bar{A} \subset \bar{E}_{n}$, one has $\mu(\bar{A}) \geq e^{-c n\left(h_{\mu}+\epsilon\right)}$, and hence the first condition of Lemma 10(I) is fulfilled (with $\gamma_{n}=c n$ ). The second condition, $(n-c n) h_{\mu}-\epsilon(n+c n)=\left((1-c) h_{\mu}-\epsilon(1+c)\right) n>0$ follows from $\epsilon<\varepsilon=\frac{(1-c)}{(1+c)} h_{\mu}$. Therefore, by Lemma 10(I),

$$
S_{2} \geq \sum_{\bar{A} \subset \bar{E}_{n}} \frac{\mu(\bar{A})}{2} e^{n\left(h_{\mu}-\epsilon\right)} \mu(\bar{A})^{s}=\frac{1}{2} e^{n\left(h_{\mu}-\epsilon\right)} \sum_{\bar{A} \subset \bar{E}_{n}} \mu(\bar{A})^{1+s}
$$

and we conclude as in 8 that $S_{2} \geq e^{n\left(h_{\mu}-\varepsilon\right)} S_{3}$, where $S_{3}=\sum_{\tilde{A} \subset \bar{E}_{n}} \mu(\tilde{A})^{1+s}$. Finally we use the fact from [8] that $S_{3} \geq e^{-2 s n \varepsilon} S_{4}$, where $S_{4}=\sum_{\tilde{A} \in \tilde{E}_{n}^{-}} \mu(\tilde{A})^{1+s}$.

We thus obtain for some constant $c_{1}$ independent of $\varepsilon$ :

$$
W_{n}^{s}(x, z) \geq e^{-c_{1} n \varepsilon} e^{n h_{\mu}} S_{4} .
$$


Lemma 14. There exists some constant $K_{14}$, which depends only on $s$, so that for almost every $x$,

$$
W_{n}^{s}(x, z) \geq e^{-K}[14]^{n \varepsilon} e^{n h_{\mu}} \sum_{\tilde{A} \in \tilde{E}_{n}^{+}} \mu(\tilde{A})^{1+s}
$$

for all $n$ large enough.

Proof. Let $\beta>1$ and define $\left(\Delta=\left[n^{\alpha}\right]\right)$

$$
\mathcal{G}_{n}=\left\{x: \mu\left(A_{n}(x)\right) \geq \exp \left(-\Delta^{\beta}\right) \mu\left(\tilde{A}_{n}(x)\right)\right\} .
$$

Then $\mathcal{G}_{n}$ is a union of $n$-cylinders, since by definition if $x \in \mathcal{G}_{n}$, we have $A_{n}(x) \subseteq \mathcal{G}_{n}$. Moreover put

$$
F_{j, \Delta}=\bigcap_{i=1}^{\Delta} T^{-i}\left(\bigcup_{m=1}^{j-1} \mathcal{P}_{m}\right)
$$

for $j \in \mathbb{N}$. Note that if $x \in F_{j, \Delta}$, then for $1 \leq i \leq \Delta, T^{i}(x) \notin \mathcal{P}_{k}$ for all $k \geq j$. The set $F_{j, \Delta}$ is a finite union of $\Delta$-cylinders and consists of point $x$ that do not hit the "tail" $\bigcup_{m=j}^{\infty} \mathcal{P}_{m}$ for the first $\Delta$ iterates. Obviously, $F_{j, \Delta} \subseteq F_{j+1, \Delta}$ and $F_{j, \Delta^{\prime}} \subseteq F_{j, \Delta}$ for $\Delta^{\prime}>\Delta$. We will consider the sets $F_{k_{n}, \Delta}$ for $k_{n}=\left[n^{t}\right], t>1$.

We make use of $\mathcal{G}_{n}$ to compare the summands $\mu\left(A_{n}\left(T^{i}(x)\right)\right)$ and $\mu\left(\tilde{A}_{n}\left(T^{i}(x)\right)\right)$ as follows:

$$
\begin{aligned}
W_{n}^{s}(x, z) & \geq \sum_{i=1}^{\tau_{n}^{z}(x)} \mu\left(A_{n}\left(T^{i}(x)\right)\right)^{s} \chi_{\mathcal{G}_{n}}\left(T^{i}(x)\right) \\
& \geq e^{-s \Delta^{\beta}} \sum_{i=1}^{\tau_{n}^{z}(x)} \mu\left(\tilde{A}_{n}\left(T^{i}(x)\right)\right)^{s} \chi_{\mathcal{G}_{n}}\left(T^{i}(x)\right) \\
& =e^{-s \Delta^{\beta}} \sum_{\tilde{A} \in \mathcal{A}^{\tilde{\gamma} n}} \mu(\tilde{A})^{s} \nu_{x}^{z}\left(\tilde{A} \cap \mathcal{G}_{n}\right) \\
& \geq e^{-s \Delta^{\beta}} \sum_{\tilde{A} \in \tilde{E}_{n}^{+}} \mu(\tilde{A})^{s} \nu_{x}^{z}\left(\tilde{A} \cap \mathcal{G}_{n}\right) .
\end{aligned}
$$

The first inequality is true since $0 \leq \chi_{\mathcal{G}_{n}} \leq 1$. The second inequality follows from the definition of $\mathcal{G}_{n}$. The last inequality is valid since we restrict the sum to a subcollection of $\tilde{A}$. In order to apply Lemma 10(II) to obtain a lower bound of $\nu_{x}^{z}\left(\tilde{A} \cap \mathcal{G}_{n}\right)$, we write

$$
\begin{aligned}
\mu\left(\tilde{A} \cap \mathcal{G}_{n}\right) & \geq \mu\left(\tilde{A} \cap T^{-\tilde{\gamma}_{n}}\left(F_{k_{n}, \Delta}\right) \cap \mathcal{G}_{n}\right) \\
& =\left[\frac{\mu\left(\tilde{A} \cap T^{-\tilde{\gamma}_{n}}\left(F_{k_{n}, \Delta}\right)\right)}{\mu(\tilde{A})}-\frac{\mu\left(\tilde{A} \cap T^{-\tilde{\gamma}_{n}}\left(F_{k_{n}, \Delta}\right) \cap \mathcal{G}_{n}^{c}\right)}{\mu(\tilde{A})}\right] \mu(\tilde{A}) .
\end{aligned}
$$

In 8 it was shown that the quantity inside the bracket goes to 1 as $n$ tends to $\infty$ (The first term converges to 1 and the second term converges to 0.) Thus $\frac{\mu\left(\tilde{A} \cap \mathcal{G}_{n}\right)}{\mu(\tilde{A})} \rightarrow 1$ as $n \rightarrow \infty$ and in particular for large enough $n, \mu\left(\tilde{A} \cap \mathcal{G}_{n}\right) \geq \mu(\tilde{A}) / 2 \geq e^{-\tilde{\gamma}_{n}\left(h_{\mu}-\epsilon\right)} / 2$ if $\tilde{A} \in \tilde{E}_{n}^{+}$. Let us now apply Lemma 10(II) where we put $\gamma_{n}=\tilde{\gamma}_{n}$. Hence

$$
\nu_{x}^{z}\left(\tilde{A} \cap \mathcal{G}_{n}\right) \geq \frac{\mu\left(\tilde{A} \cap \mathcal{G}_{n}\right)}{2} e^{n\left(h_{\mu}-\epsilon\right)} \geq \frac{\mu(\tilde{A})}{4} e^{n\left(h_{\mu}-\epsilon\right)} .
$$

From (10) one thus obtains

$$
W_{n}^{s}(x, z) \geq \frac{\exp \left(-s \Delta^{\beta}\right)}{4} \exp (-n \epsilon) \exp \left(n h_{\mu}\right) \sum_{\tilde{A} \in \tilde{E}_{n}^{+}} \mu(\tilde{A})^{1+s} .
$$

Now let $\beta \in(1,1 / \alpha)$ so that $\alpha \beta<1$, then $n$ dominates $\Delta^{\beta}=\left[n^{\alpha}\right]^{\beta}$ and hence there exists $K[14$ so that for large enough $n, e^{-n \epsilon} e^{-s \Delta^{\beta}} / 4 \geq e^{-K}\left[\left[^{n \varepsilon}\right.\right.$. 


\section{Acknowledgment}

Part of this research was carried out during a visit of the fourth named author to the Mathematics Department at the University of Southern California, Los Angeles, in 2012. She thanks this department for its hospitality.

\section{References}

[1] M. Abadi: Sharp Error Terms and Neccessary Conditions for Exponential Hitting Times in Mixing Processes; Ann. of Probab. 32 (2004), 243-264.

[2] L. Carleston: Two remarks on the basic theorems of information theory; Math. Scand. 6 (1958), $175-180$.

[3] K. Chung: A note of ergodic theorem of information; Ann. of Math. Statist. 32 (1961), 612-614.

[4] V. M. Deschamps, B. Schmitt, M. Urbanski and A. Zdunik: Pressure and Recurrence; Fund. Math. 178 (2003), 129-141.

[5] T. Downarowicz: Entropy in Dynamical Systems; Cambridge University Press 2011.

[6] N. Haydn and S. Vaienti: The Rényi Entropy Function and the Large Deviation of Short Return Times; Ergodic Th. \& Dyn. Sys. 39 (2010), 159-179.

[7] N. Haydn, Y. Lacroix and S. Vaienti: Hitting and Return Times in Ergodic Dynamical Systems, Ann. of Probab. 33 (2005), 2043-2050.

[8] M. Ko: Rényi Entropy and Recurrence; to appear in Disc. Cont. Dynam. Syst. 2012.

[9] R. Mañé: Ergodic Theory and Differentiable Dynamics; Springer 1987.

[10] D. Ornstein and B. Weiss: Entropy and Data Compression schemes; IEEE Trans. Inf. Theory 39 (1993), 78-83.

[11] D. Ornstein and B. Weiss: Entropy and Recurrence Rates for Stationary Random Fields; IEEE Trans. Inf. Theory 48(6) (2002), 1694-97.

[12] K. Petersen: Ergodic Theory; Cambridge studies in advanced mathematics 2, 1983.

[13] A. Rényi: Probability Theory; North Holland, Amsterdam 1970.

[14] C. Shannon: A Mathematical Theory of Communication; The Bell System Technical Journal 27 (1948) 379-423, 623-656.

[15] P. Shields: Waiting Times: Positive and Negative Results on the Wyner-Ziv Problem; Theoretical Prob., Vol. 6, No. 3, 1992. 